\title{
Neural Correlates of Alcohol-Approach Bias in Alcohol Addiction: the Spirit is Willing but the Flesh is Weak for Spirits
}

\author{
Corinde E Wiers* ${ }^{* 1,2,3}$, Christine Stelzel',2,4, Soyoung Q Park ${ }^{5}$, Christiane K Gawron', Vera U Ludwig ${ }^{1,2,4}$, \\ Stefan Gutwinski ${ }^{1,2}$, Andreas Heinz ${ }^{1,2,6}$, Johannes Lindenmeyer ${ }^{7,8}$, Reinout W Wiers', Henrik Walter ${ }^{1,2,6,10}$ \\ and Felix Bermpohl ${ }^{1,2,6,10}$
}

'Department of Psychiatry and Psychotherapy, Charité - Universitätsmedizin Berlin, Berlin, Germany; ${ }^{2}$ Berlin School of Mind and Brain, Humboldt University Berlin, Berlin, Germany; ${ }^{3}$ Department of Education and Psychology, Free University Berlin, Berlin, Germany; ${ }^{4}$ Department of Psychology, Humboldt University Berlin, Berlin, Germany; ${ }^{5}$ Department of Economics, University of Zürich, Zürich, Switzerland; 'Berlin Center for Advanced Neuroimaging, Charité - Universitätsmedizin Berlin, Berlin, Germany; ${ }^{7}$ Salus Klinik, Lindow, Germany; ${ }^{8}$ Department of Clinical Psychology, Chemnitz University of Technology, Chemnitz, Germany; ${ }^{9}$ Addiction, Development and Psychopathology (ADAPT) Lab, Department of Psychology, University of Amsterdam, Amsterdam, The Netherlands

\begin{abstract}
Behavioral studies have shown an alcohol-approach bias in alcohol-dependent patients: the automatic tendency to faster approach than avoid alcohol compared with neutral cues, which has been associated with craving and relapse. Although this is a well-studied psychological phenomenon, little is known about the brain processes underlying automatic action tendencies in addiction. We examined 20 alcohol-dependent patients and 17 healthy controls with functional magnetic resonance imaging (fMRI), while performing an implicit approach-avoidance task. Participants pushed and pulled pictorial cues of alcohol and soft-drink beverages, according to a contentirrelevant feature of the cue (landscape/portrait). The critical fMRI contrast regarding the alcohol-approach bias was defined as (approach alcohol>avoid alcohol)> (approach soft drink>avoid soft drink). This was reversed for the avoid-alcohol contrast: (avoid alcohol > approach alcohol) > (avoid soft drink > approach soft drink). In comparison with healthy controls, alcohol-dependent patients had stronger behavioral approach tendencies for alcohol cues than for soft-drink cues. In the approach, alcohol fMRI contrast patients showed larger blood-oxygen-level-dependent responses in the nucleus accumbens and medial prefrontal cortex, regions involved in reward and motivational processing. In alcohol-dependent patients, alcohol-craving scores were positively correlated with activity in the amygdala for the approach-alcohol contrast. The dorsolateral prefrontal cortex was not activated in the avoid-alcohol contrast in patients vs controls. Our data suggest that brain regions that have a key role in reward and motivation are associated with the automatic alcoholapproach bias in alcohol-dependent patients.
\end{abstract}

Neuropsychopharmacology (2014) 39, 688-697; doi:I0.1038/npp.2013.252; published online 16 October 2013

Keywords: addiction; approach bias; alcoholism; $\mathrm{MMR}$; reward; craving

\section{INTRODUCTION}

Addiction is characterized by habitual drug use, despite negative consequences, and by high rates of relapse, even though the addicted person is often aware of the harm (Stacy and Wiers, 2010). Recent theories suggest rewardrelated learning to be important for the development of addiction (Hyman et al, 2006; Wrase et al, 2002): a transition occurs from voluntary to impulsive use, in which cues associated with the drug increase in incentive salience

*Correspondence: CE Wiers, Berlin School of Mind and Brain, Humboldt University Berlin, Unter den Linden 6, 10099 Berlin, Germany, Tel: + 49302093 6190, Fax: +49302093 1802,

E-mail: corinde.wiers@gmail.com

${ }^{10}$ These authors contributed equally to this work.

Received 19 April 2013; revised 24 July 20 I3; accepted 26 July 20 I3; accepted article preview online 20 September 2013
(Robinson and Berridge, 1993, 2003). Drug cues then automatically trigger drug-like approach responses (Robinson and Berridge, 1993, 2003). Dual process models of addiction propose an imbalance between these strong automatic 'approach'-oriented processes and a suboptimal functioning of cognitive control processes (Bechara, 2005; Gladwin et al, 2011). This imbalance may explain the paradoxical conflict that characterizes addiction: urges to take the drug that the individual fails to control despite an explicit desire to quit.

Previous research has demonstrated that drug-dependent individuals exhibit an automatically activated tendency to approach rather than to avoid drug cues relative to neutral cues (ie, drug approach bias; Cousijn et al, 2011; Ernst et al, 2012; Wiers et al, 2013; Zhou et al, 2012). The drug approach bias is likely to reflect an embodied motor reaction towards drug cues and has been positively related 
to subjective rates of drug craving (Wiers et al, 2013). Moreover, bias modification training schemes, in which drug users learn to avoid drug cues in a joystick paradigm, have been shown to reduce relapse rates up to $13 \%$ in alcohol-dependent patients 1 year after training (Eberl et al, 2012; Wiers et al, 2011). These findings highlight the clinical relevance of approach bias in drug use. However, neural correlates associated with the drug approach bias remain largely unknown.

The incentive sensitization theory of addiction suggests fronto-limbic neuroadaptations to underlie the drug approach bias (Robinson and Berridge, 2003). Many drugs of abuse (eg, alcohol, nicotine, or cocaine) directly or indirectly trigger the release of dopamine from the ventral tegmental area, projecting to fronto-limbic structures such as the nucleus accumbens (NAcc) and medial prefrontal cortex (mPFC; Heinz et al, 2009; Hyman and Malenka, 2001). As dopamine signals motivational relevance with every puff, drink, or shot, Pavlovian-conditioned associations between drug cues and reward are formed and encoded in the amygdala (Baler and Volkow, 2006; Heinz et al, 2009). In this way, drug cues acquire incentive sensitization and consequently engender approach behavior (Robinson and Berridge, 1993, 2003).

Human functional magnetic resonance imaging (fMRI) studies have shown that when drug users passively view drug cues, blood-oxygen-level-dependent (BOLD) signal (hereafter referred to as activity) in the fronto-limbic reward circuit increases (Heinz et al, 2009; Schacht et al, 2013). Key brain areas that activate in drug users are the NAcc, mPFC, dorsolateral prefrontal cortex (dlPFC), and amygdala (Heinz et al, 2009; Schacht et al, 2013). However, despite the evidence for fronto-limbic involvement in drugcue reactivity, the precise role of these areas remains unclear. The NAcc, mPFC, and amygdala have been associated with bottom-up motivational aspects of cue reactivity (Braus et al, 2001; Hare et al, 2009; Heinz et al, 2009; Wrase et al, 2007), reward processing (Kahnt et al, 2010; Koob and Volkow, 2010; Park et al, 2011), subjective drug craving, and relapse (Beck et al, 2012; Childress et al, 1999; Grusser et al, 2004; Hayashi et al, 2013; Heinz et al, 2004; Volkow et al, 2004). The dlPFC has been shown to be structurally and functionally impaired in drug addiction, and may be related to suboptimal cognitive control (Baler and Volkow, 2006; Bechara, 2005; Hayashi et al, 2013; Jentsch and Taylor, 1999; Park et al, 2010). Previous approach/avoidance studies on emotional processing showed that dIPFC is more active when stimulus and response are incongruent (approach sad faces) than congruent (approach happy faces; Roelofs et al, 2009; Volman et al, 2011). If patients indeed have an alcoholapproach bias (congruent), the dlPFC would be expected to be active while avoiding alcohol cues (incongruent). Alternatively, when patients lack the control to avoid alcohol cues, one would expect decreased dlPFC activation for avoiding alcohol cues. How these antagonistic processes of motivation and control underlie automatic approach tendencies for alcohol as yet remains unknown.

In the current study, we measured the neural correlates of the automatic alcohol-approach bias using fMRI. Abstinent alcohol-dependent patients and healthy controls performed an implicit approach-avoidance task (AAT) in an fMRI scanner. As such, this is the first study that investigates the neural correlates of the alcohol-approach bias in alcohol dependence using fMRI. Participants pushed and pulled pictorial cues of alcohol and soft-drink beverages using a joystick. Compared with controls, patients were hypothesized to faster pull than push alcohol stimuli compared with soft-drink stimuli. For the fMRI alcohol-approach bias interaction (approach alcohol $>$ avoid alcohol) $>$ (approach soft drink>avoid soft drink), we expected increased activity in the NAcc, mPFC, and amygdala, areas previously associated with reward and motivational processing. Subjective craving scores of alcohol-dependent patients were hypothesized to correlate positively with the alcoholapproach bias-related activity in these regions. Lastly, we investigated whether patients showed either greater or reduced dlPFC activity than healthy controls in a reverse avoid-alcohol contrast (avoid alcohol $>$ approach alcoho1) $>$ (avoid soft drink $>$ approach soft drink), indicating enhanced or reduced inhibitory control, respectively.

\section{MATERIALS AND METHODS}

\section{Participants and Instruments}

The Ethical Committee of the Charité, Universitätsmedizin Berlin, approved the study. Thirty-seven right-handed male subjects participated: 20 alcohol-dependent in-patients $(M=44.3$ years $(\mathrm{SD}=7.98)$, range $=26-55)$ and 17 healthy control subjects $(M=42.1, \mathrm{SD}=8.32$, range $=22-53)$. The groups did not differ in mean age and years of education (Table 1). Controls were recruited via online advertisements. Exclusion criteria for all participants were a history of neurological dysfunctions, axis I psychiatric disorders according to DSM-IV criteria other than alcohol dependence in the alcohol-dependent group (M.I.N.I. plus, an International Neuropsychiatric Interview; Sheehan et al, 1998), and intake of psychoactive medication. Controls did not fulfill criteria of (a history of) drug abuse and dependence, except tobacco. For controls, potential participants with scores above 8 on the Alcohol Use Disorder Identification Test (Saunders et al, 1993) were excluded, as screened in a telephone interview before the experiment. Patients were recently detoxified ( $<6$ months; $M=53.40$ days, $S D=49.51)$, had been suffering from alcohol dependence for $16.6(\mathrm{SD}=8.5$ years, range $1-30)$, underwent 3.9 $(\mathrm{SD}=6.7)$ previous detoxifications (range $0-25)$, and scored $16.4(\mathrm{SD}=8.4)$ on the Alcohol Dependence Scale (Skinner and Allen, 1982). Smokers were abstinent from tobacco at least $1.5 \mathrm{~h}$ before scanning, in order to decrease direct effects of nicotine on the BOLD signal (Jacobsen et al, 2002). All patients expressed the desire to remain abstinent from alcohol.

In order to assess lifetime history of alcohol and drug abuse for both groups, we interviewed participants on the Life Time Drinking History scale (Skinner and Sheu, 1982). Alcohol craving was assessed with the Desire for Alcohol Questionnaire (DAQ; Love et al, 1998). Furthermore, participants completed Matrix Reasoning of the Wechsler Adult Intelligence scale (Kaufman and Lichtenberger, 2006) as a proxy for general intelligence and the Spielberger's State-Trait Anxiety Questionnaire (STAI) to evaluate state and trait anxiety (Spielberger et al, 1983). 
Table I Demographic and Clinical Data of Alcohol-Dependent Patients and Healthy Controls

\begin{tabular}{|c|c|c|c|}
\hline & Alcohol-dependent patients $(n=20$, all male) & Healthy controls $(n=17$, all male) & $P$-value \\
\hline Age (years) & $44.30(7.98)$ & $42.12(8.3)$ & 0.422 NS \\
\hline BMI & $23.98(3.43)$ & $24.44(2.33)$ & 0.637 NS \\
\hline Years of education & $10.55(1.15)$ & $11.29(1.72)$ & $0.141 \mathrm{NS}$ \\
\hline WAIS matrices score & |5.2| (4.43) & $17.24(6.13)$ & $0.260 \mathrm{NS}$ \\
\hline STAI-T & $36.00(8.30)$ & $34.24(12.09)$ & $0.630 \mathrm{NS}$ \\
\hline Lifetime alcohol intake (kg) & $2052.74(2821.01)$ & $153.62(225.92)^{\mathrm{a}}$ & $0.007^{* * * *}$ \\
\hline AUDIT & $27.05(7.82)$ & $2.82(1.67)$ & $0.000^{* * *}$ \\
\hline ADS & $16.43(8.04)^{b}$ & - & - \\
\hline Abstinence (days) & $53.40(49.5 I)$ & - & - \\
\hline
\end{tabular}

Abbreviations: ADS, Alcohol Dependence Scale; AUDIT, Alcohol Use Disorders Test; BMI, body mass index; DAQ, Desire for Alcohol Questionnaire; NS, not significant; STAI-S, State Anxiety Inventory; STAI-T, Trait Anxiety Inventory; WAIS, Wechsler Adult Intelligence Scale.

$p<0.001$

${ }^{\mathrm{a}} \mathrm{N}=16$.

${ }^{\mathrm{b}} \mathrm{N}=19$.

\section{AAT Description and Subjective Rating}

A zoom version of the AAT, optimized for MRI, was used (Figure 1). Participants pushed or pulled an MRI-compatible joystick (Fiber Optic Joystick, Current Designs), in response to the format of the cue (landscape or portrait). After 20 practice trials, 160 test trials were presented over 4 blocks, in which each picture was approached and avoided once. Picture format to response assignment was counterbalanced, with half of the participants pulling the joystick for landscape and pushing it for portrait cues, and vice versa. For optimal approach and avoidance resemblance (Rinck and Becker, 2007), the AAT used here was developed with a zooming feature: moving the joystick increased and decreased the size of the cue. Participants had to respond to a picture within $2 \mathrm{~s}$. Intertrial intervals were 4,6 , or $8 \mathrm{~s}$ distributed hyperbolically (Miezin et al, 2000). A set of 40 alcohol and 40 soft-drink images was used, previously matched for drink familiarity and for arousal in an independent male, social drinking German sample $(N=20)$. Images $(660 \times 660$ pixels $)$ were presented in a white frame $(900 \times 660$ pixels landscape and $660 \times 900$ pixels portrait format), against a black background. The task was programmed in MATLAB (r2010a; MathWorks Company) and Psychtoolbox v3 (Brainard, 1997). After scanning, all pictures were rated for familiarity ('How familiar is this drink to you?'), arousal ('How much does this drink move you?'), and valence ('How positive or negative is this drink to you?'), on a five-point Likert scale.

\section{fMRI Acquisition and Preprocessing}

Stimuli were presented in an event-related design (4 runs of 40 trials) in a $3 \mathrm{~T}$ MRI scanner (MAGNETOM Trio, TIMTechnology; Siemens, Erlangen, Germany), equipped with a

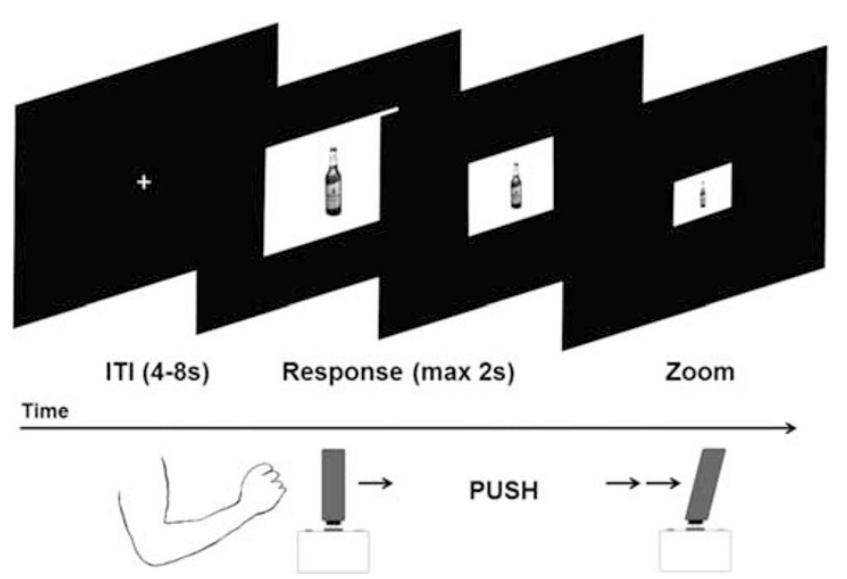

Figure I Schematic overview of an avoid alcohol trial on the approachavoidance task (AAT), in which the cue zooms out while pushing on the joystick.

12-channel head coil. A standard T2-weighted echo planar imaging (EPI) sequence was used with the following parameters: descending, repetition time $2 \mathrm{~s}$, echo time $25 \mathrm{~ms}$, flip angle $\alpha 80^{\circ}, 64 \times 64$ pixels in-plane resolution, 34 slices, slice thickness $3 \mathrm{~mm}$, voxel dimensions $3 \times 3 \times 3 \mathrm{~mm}^{3}$, with a $0.75-\mathrm{mm}$ gap between slides, and field of view $192 \times$ $192 \mathrm{~mm}^{2}$. In each of the 4 runs, 163 images were acquired. To improve functional sensitivity in the MPFC, the acquisition plane was tilted $25^{\circ}$ clockwise from anterior-posterior commissure (Deichmann et al, 2003).

Functional data analysis was performed with SPM8 (Wellcome Department of Cognitive Neurology, London, UK). During preprocessing, scans were spatially realigned, slice-time corrected and normalized to the standard 
Montreal Neurological Institute (MNI) EPI template. Smoothing was performed with an $8-\mathrm{mm}$ full-width at half-maximum Gaussian kernel. None of the participants moved more than $2 \mathrm{~mm}$ or $2^{\circ}$ within runs.

\section{Statistical Analysis}

Responses that were missed or incorrect and response times (RTs) longer than 3 SDs above the mean were discarded based on each participant's performance. RTs were computed as the time required from the onset of stimulus presentation until the joystick reached a maximum or minimum position. Approach tendencies were calculated by subtracting median RT scores of pushing minus pulling pictures for each drink type. Positive approach tendencies indicate faster approaching than avoiding an image type, whereas negative approach tendencies indicate faster avoidance than approach. A $2 \times 2$ mixed ANOVA on approach tendencies was calculated, with drink type (alcohol/soft drink) as the within-subject factor and group (alcohol-dependent /healthy control) as the between-subject factor. Post-hoc group comparisons on separate approach tendencies (alcohol/soft drink) were performed with twosided two-sample Student's $t$-tests. The behavioral alcoholapproach bias RT score was defined as the difference score of approach tendency for alcohol minus approach tendency for soft drink. Pearson's correlation was calculated between the behavioral alcohol-approach bias and DAQ alcohol craving.

For fMRI data, there were five regressors per subject: alcohol push, alcohol pull, soft-drink push, soft-drink pull, and missed trials. Single trials were modeled with the trial's RT as duration of the event and convolved with the hemodynamic response function. The six realignment parameters were included as regressors of no interest. Temporal filtering of $128 \mathrm{~s}$ was used.

The following contrasts were calculated per subject: (1) (approach alcohol $>$ avoid alcohol) $>$ (approach soft drink $>$ avoid soft drink) for the approach-alcohol contrast and (2) the reverse (avoid alcohol $>$ approach alcohol) $>$ (avoid soft drink>approach soft drink) for the avoid-alcohol contrast. On the second level, both contrasts were compared between groups using a two-sample Student's $t$-test. We created four regions of interest (ROIs), based on our a priori hypotheses (Figure 2). Both the NAcc and amygdala ROIs were defined by the bilateral NAcc and amygdala using the human anatomical WFU Pickatlas (Maldjian et al, 2003). As $\mathrm{mPFC}$ and bilateral dlPFC are anatomically not clearly defined, two functional ROIs of these brain areas were downloaded from an online atlas of functional ROIs (Shirer et al, 2012). ROIs were used for small-volume correction (SVC) of the results, with a significance threshold of $p<0.05$, family-wise error corrected (FWE).

For post-hoc analyses, two approach tendency contrasts were calculated on the first level: (1) (approach alcohol> avoid alcohol) and (2) (approach soft drink $>$ avoid soft drink). These contrasts were compared between groups using two-sample Student's $t$-tests, masked with our a priori defined ROIs.

To test whether length of abstinence was negatively related to activity in our ROIs, regression analyses within
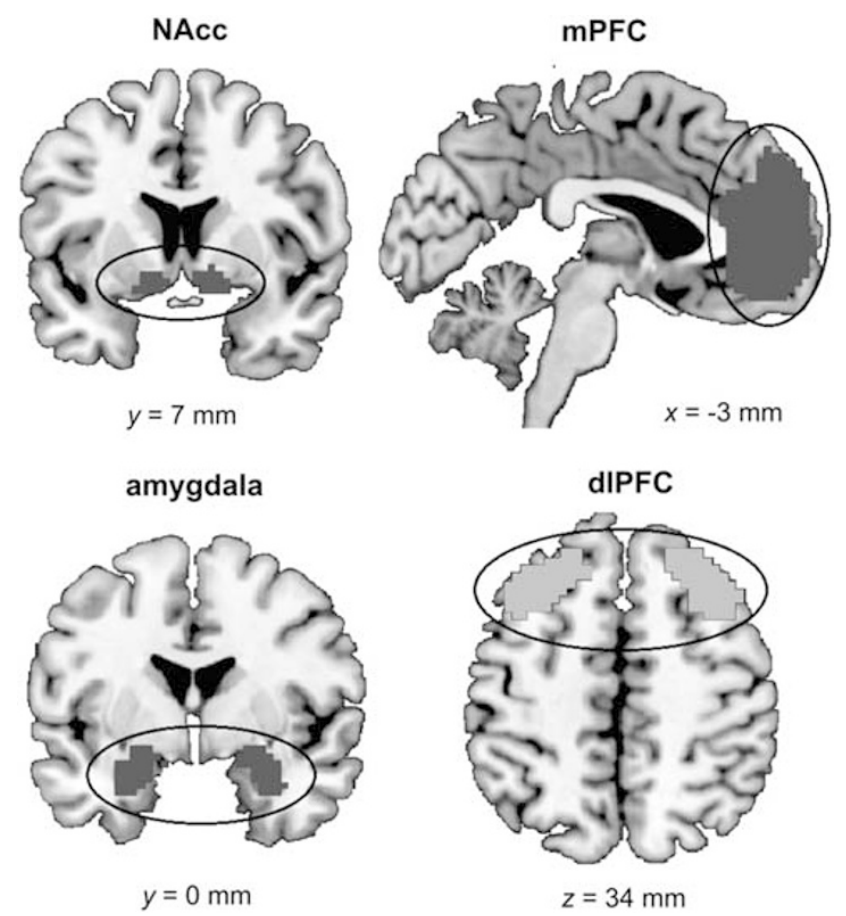

Figure 2 A-priori defined regions of interests of the motivational system (nucleus accumbens (NAcc), medial prefrontal cortex (mPFC), and amygdala) and of the cognitive control system (dorsolateral prefrontal cortex (dIPFC)), shown in red and blue respectively.

these ROIs with length of abstinence as a regressor were performed in alcohol-dependent patients only.

To identify correlations with DAQ craving scores and alcohol-approach bias-related brain activations in alcoholdependent patients, we performed a regression analysis on the approach-alcohol contrast, with DAQ scores as a regressor. Results of correlations were FWE corrected (SVC) for our ROIs (NAcc, mPFC, and amygdala).

\section{RESULTS}

\section{Behavioral Assessment and Subjective Ratings}

Groups did not differ in years of education, body mass index, Wechsler Adult Intelligence Scale intelligence scores, or in anxiety trait (STAI-T) and state (STAI-S) scores (Table 1). There were more smokers in the alcoholdependent group $(N=20,100 \%)$ compared with the control group $\left(N=6,28.3 \% ; \chi^{2}=7.54, p=0.006\right)$. However, we did not include smoking as a covariate, as smoking behavior was related to lifetime alcohol consumption $(R=0.58$, $p=0.007$; also when corrected for age: $R=0.58, p=0.007)$ and DAQ craving scores $(R=0.45, p=0.047)$ in alcoholdependent patients. Consequently, including smoking as a covariate may remove variance explained by drinking behavior (an exploratory analysis with smoking behavior as a covariate in the main group analysis revealed that whereas results on the NAcc $(p<0.05$, FWE) and dlPFC (lack of effect at $p>0.05$, FWE) in both the approach and avoid contrast did not change, activity in the mPFC did not reach significance when including smoking as a covariate $(p>0.05, \mathrm{FWE}))$. 


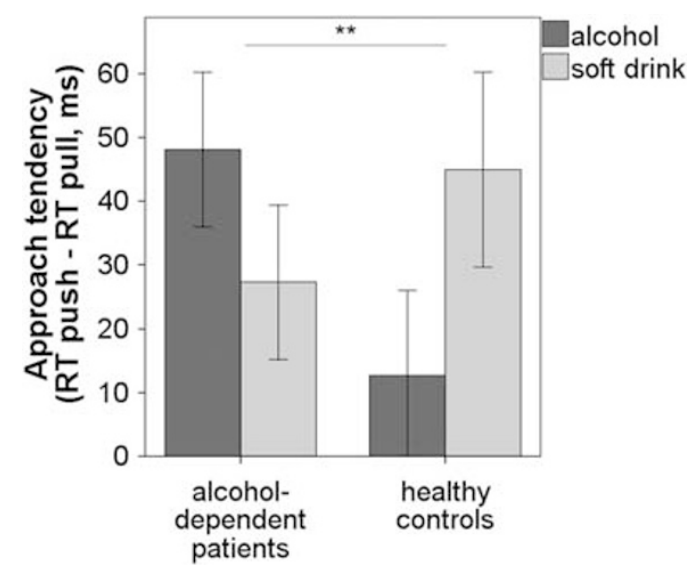

Figure 3 Mean approach tendencies (response time (RT) avoidapproach) for alcohol and soft-drink cues. There was a significant interaction effect of drink type $\times$ group $(p<0.0 I)$, with alcohol cues being approached faster in alcohol-dependent patients as a trend $(p<0.06)$. Error bars depict I SE above and below the mean. ${ }^{* *} p<0.01$.

DAQ alcohol-craving ratings were higher in alcoholdependent patients $(M=14.3, \mathrm{SD}=6.7)$ compared with healthy controls $(M=4.88, \quad \mathrm{SD}=4.4 ; \quad t(35)=3.55$, $p=0.001$ ). Picture ratings (familiarity, valence, and arousal) for both alcohol and soft-drink cues did not differ between groups (all $p>0.13$ ).

For the AAT, all RT variables, both separate approach tendencies (alcohol/soft drink) and the overall alcoholapproach bias score were distributed normally (KolmogorovSmirnov test: all $p>0.12$ ). The assumption of homogeneity of variance was met in all cases (Levene's test for equality of variance: all $p>0.52)$. Mean error rate was $1.71 \%(\mathrm{SD}=1.95)$ and error rates did not differ between groups $(t(35)=0.49$, $p=0.63$ ).

As hypothesized, for behavioral approach tendencies there was a significant interaction effect between drink type $\times$ group $\left(F(1,34)=9.99, p=0.003, \eta^{2}=0.22\right.$; Figure 3$)$. Post-hoc Student's $t$-tests revealed that patients had greater approach tendencies for alcohol cues $(M=48.10 \mathrm{~ms}$, $\mathrm{SD}=54.35)$ compared with healthy controls by trend $(M=12.71 \mathrm{~ms}, \mathrm{SD}=54.61 ; t(35)=1.97, p=0.057)$. In contrast, approach tendencies for soft-drink cues did not differ between groups $(t(35)=-0.92, p=0.37)$.

The behavioral alcohol-approach bias did not correlate with DAQ alcohol-craving scores, either in alcohol-dependent patients $(r=0.17, p=0.48)$ or in healthy controls $(r=-0.18, p=0.49)$.

\section{fMRI Results}

Approach alcohol. For the main contrast of interest (approach alcohol $>$ avoid alcohol) $>$ (approach soft drink $>$ avoid soft drink), alcohol-dependent patients showed a higher BOLD response in the NAcc area (peak in MNI space $[x, y, z]=[15,5,-8]) ; t=3.54, p<0.05$, FWE) and the mPFC (peak $=[0,59,7]) ; t=4.43, p<0.05, \mathrm{FWE})$ compared with that in healthy controls (Figure 4). The amygdala was not more strongly activated in patients compared with controls (bilateral; $p>0.05$, FWE), even at a more liberal threshold of $p<0.005$ uncorrected. Although the dlPFC was not more activated in patients than in controls with a threshold of $p<0.05$, FWE, a cluster in the left dlPFC survived the exploratory, a more liberal threshold of $p<0.005$ uncorrected.

Post-hoc Student's $t$-tests on separate approach tendency contrasts revealed that alcohol-related activity (approach alcohol $>$ avoid alcohol) did not differ between groups in the NAcc and mPFC ( $p>0.005$ uncorrected). Approach tendency-related activity for soft-drink cues (approach soft drink > avoid soft drink) was larger in healthy controls than in alcohol-dependent patients, both in the NAcc (peak $=[9,8,-8] ; t=3.08, \quad p<0.05, \quad F W E)$ and $\mathrm{mPFC}$ $($ peak $=[-12,56,7]) ; t=5.15, p<0.05, \mathrm{FWE})$.

Within patients, length of abstinence was not correlated with the approach-alcohol contrast within our ROIs ( $p>0.05$, few, and $p>0.005$, uncorrected).

Avoid alcohol. In the reverse avoid-alcohol contrast (avoid alcohol $>$ approach alcohol) $>$ (avoid soft drink $>$ approach soft drink), no suprathreshold activity was reached in the dlPFC (bilateral; $p>0.05$, FWE) in patients versus controls. Moreover, an additional analysis with a more liberal threshold of $p<0.005$ (uncorrected) did not reveal suprathreshold activity.

Correlation of craving scores with alcohol-approach bias-related activity in alcohol-dependent patients. Alcoholdependent patients' DAQ craving scores correlated positively with activity in the amygdala for the approach-alcohol contrast (peak $=[30,-7,-11]$ ); $t=4.25, p<0.05$, FWE; Figure 5). There were no positive correlations between alcohol craving and activity in the NAcc or MPFC, even at $p<0.005$ uncorrected.

Results of whole brain-analyses are reported in Supplementary Table S1.

\section{DISCUSSION}

The current study shows that in comparison with healthy controls, alcohol-dependent patients had stronger behavioral approach tendencies for alcohol cues than for softdrink cues. At the neural level, the alcohol-approach bias interaction of drink type (alcohol $v s$ soft drinks) $\times$ movement (approach $v s$ avoid) was associated with stronger brain response in both the NAcc and $\mathrm{mPFC}$, areas that have previously been shown to have a role in alcohol cue reactivity, reward processing, and the motivational value of stimuli (Grusser et al, 2004; Hare et al, 2009; Heinz et al, 2009; Kahnt et al, 2010; Park et al, 2011). Here we show that these areas are more active in patients $v s$ controls while approaching $v s$ avoiding alcohol cues, relative to soft-drink cues. This extends previous studies, which mostly involved passive viewing of alcohol cues (Heinz et al, 2009; Schacht et al, 2013). However, no strong effects were found in the dlPFC. Thus, we did not find direct support for enhanced or decreased neural inhibitory control, while patients were avoiding alcohol. The results suggest that differences in the motivational reward system, rather than a less active control system, underlie automatic action tendencies to alcohol in alcohol dependence.

The main findings support incentive sensitization models of addiction that propose fronto-limbic neuroadaptations to 
a
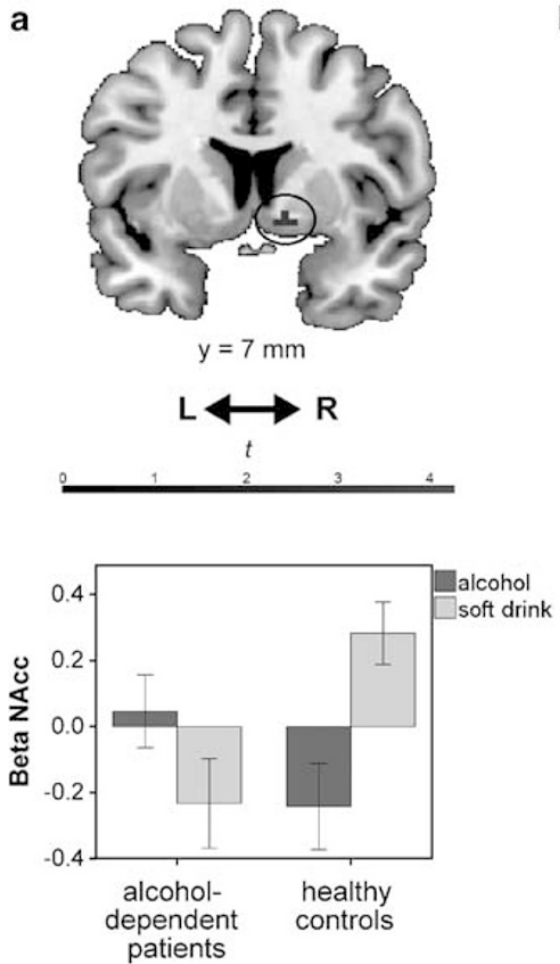

b
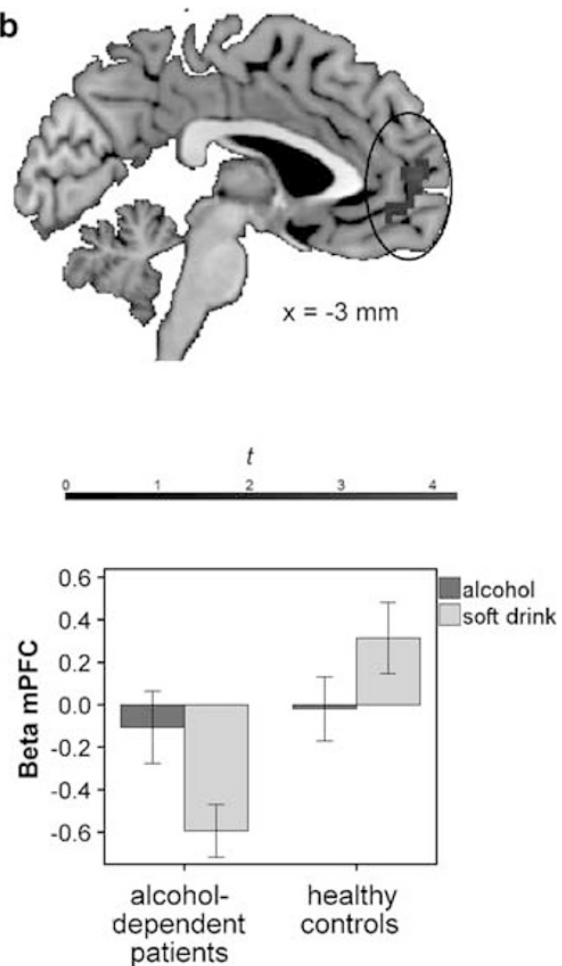

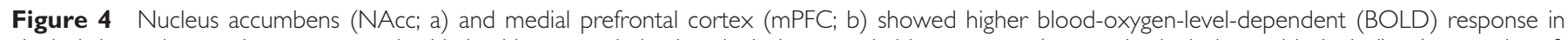

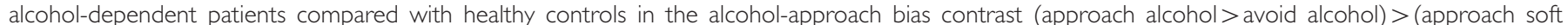

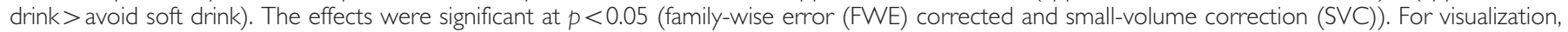
activations within our NAcc regions of interest (ROl; a) and our mPFC ROI (b) are plotted with a threshold of $p<0.005$ uncorrected, on a standard

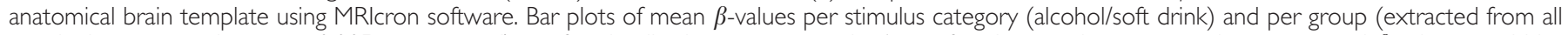
voxels that were active at $p<0.005$ uncorrected) are for visualization purposes only. As performing post-hoc tests on these extracted $\beta$-values would be

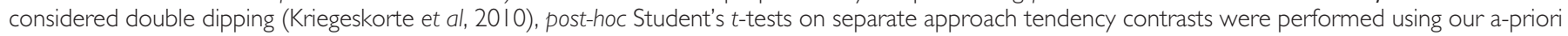

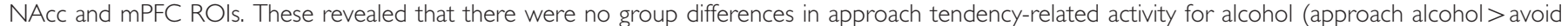

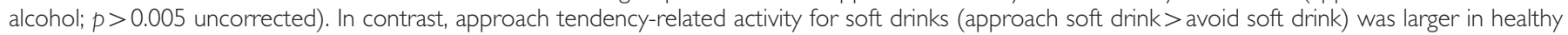
controls than alcohol-dependent patients, both in the NAcc $(p<0.05$, FWE) and mPFC ( $p<0.05$, FWE).

underlie the automatic approach bias to drug cues in addicted individuals (Robinson and Berridge, 1993, 2003). The NAcc has been shown to be responsive to alcohol cue reactivity in alcohol-dependent patients (Braus et al, 2001; Heinz et al, 2009; Wrase et al, 2007) and regulates drug sensitization in animals (Abrahao et al, 2011). The mPFC is hypothesized to code subjective value signals important for goal-directed decision making (Hare et al, 2009; Kahnt et al, 2010; Park et al, 2011) and has been related to the attribution of incentive salience to alcohol cues (Grusser et al, 2004). Recently, activity in the MPFC was shown for the cannabis approach bias in both cannabis users and nonsmoking controls (Cousijn et al, 2012). Moreover, a recent near-infrared spectroscopy study demonstrated that the neighboring orbitofrontal cortex is active when alcoholdependent patients approach alcohol cues (Ernst et al, 2012). Hence, the NAcc and mPFC may have important roles in the drug approach bias.

As expected, alcohol-dependent patients reported higher subjective craving for alcohol when compared with the control group. Although the amygdala was not activated in the main approach-alcohol contrast in patients $v s$ controls, alcohol-approach bias-related brain activity in the amygdala correlated positively with alcohol-craving scores in patients. This finding is in line with previous neuroimaging findings that also showed a positive relation between activity in the amygdala while passively viewing alcohol cues and subjective craving (Childress et al, 1999; Koob and Volkow, 2010). The amygdala has a key role in Pavlovian-conditioned learning, and the formation and consolidation of emotional memories (Koob and Volkow, 2010; Volkow et al, 2004). Drug craving may therefore lead to increased memories of the abused drug or, in reverse, approaching alcohol may trigger drug associations and memories that initiate craving. However, our results did not support the hypotheses that increased NAcc and MPFC were related to subjective craving in patients. It may therefore be that NAcc and $\mathrm{mPFC}$ are specific for automatic approach reactions rather than explicit subjective judgments of drug craving, whereas the amygdala is only activated in patients that are explicitly aware of their craving.

The dlPFC was neither more nor less active in our avoidalcohol contrast in patients $v s$ controls, under the stringent threshold of $p<0.05$, FWE. More activity in the dlPFC was expected in patients compared with controls, as previous studies found that the dlPFC is generally more active when stimulus and response are incongruent (Roelofs et al, 2009; Volman et al, 2011). In contrast, it may be that patients lack control to avoid alcohol and hence show reduced dlPFC activity in the avoid-alcohol contrast. In the latter contrast, 


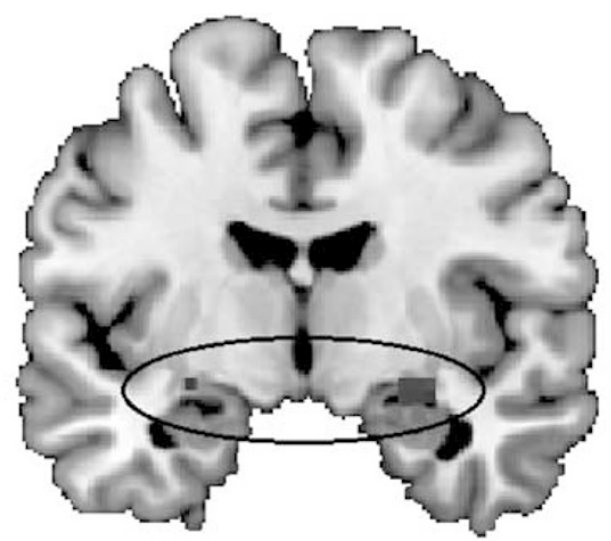

$y=-7 \mathrm{~mm}$

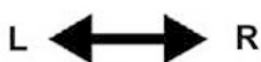

$t$
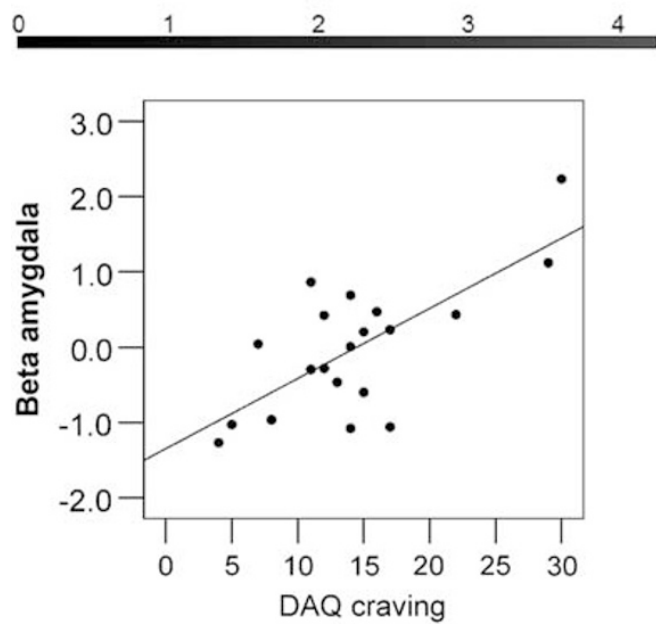

Figure 5 Craving scores correlated positively with alcohol-approach bias-related brain activity in the amygdala in alcohol-dependent patients $(p<0.05$, family-wise error (few) corrected and small-volume correction $(\mathrm{SVC})$ ). Activations within the amygdala regions of interest (ROI) are plotted here on a standard anatomical brain template using MRIcron software, with a threshold of $p<0.005$ uncorrected. Mean $\beta$-values were extracted from the activated clusters within the amygdala ROI (at $p<0.005$ uncorrected), in order to produce the correlation plot. The correlation plot is for visualization purposes only. No further post-hoc tests were performed on these extracted data, as this would be considered double dipping (Kriegeskorte et al, 20 I0).

a cluster in the left dlPFC survived the exploratory, more liberal threshold of $p<0.005$ uncorrected. As this result is uncorrected only, it cannot be firmly interpreted. Thus, differences in dlPFC activation between groups cannot be excluded at this point (and neither can they be confirmed). As alcohol-dependent patients were all clinic in-patients, it may be that avoiding alcohol cues was not incongruent for this population. This could explain why the dlPFC was not activated in the avoid-alcohol contrast in patients compared with controls. Future studies could investigate whether neural correlates of the alcohol-approach bias in social or hazardous drinkers for whom drinking is not (yet) problematic would involve dlPFC activity for avoiding alcohol. Moreover, in high-risk cannabis smokers, higher dlPFC activity during cannabis approach trials but lower activity during cannabis avoidance trials were associated with decreases in cannabis problem severity 6 months later (Cousijn et al, 2012). Although these findings were obtained with a structurally different task, in which participants symbolically approach the drug in certain mini-blocks and avoid it in other mini-blocks, future studies could focus on alcohol-approach bias-related dlPFC activity in relation to future addiction severity in alcohol dependence.

Post-hoc Student's $t$-tests revealed that the significant behavioral alcohol-approach bias interaction was mainly driven by a trend-wise difference between groups in approach tendencies for alcohol cues rather than soft-drink cues. That is, as hypothesized, behavioral approach tendencies for alcohol were larger in patients $v s$ controls, whereas there was no detectable group difference for soft-drink approach tendencies. In contrast, the interaction of BOLD responses in mPFC and NAcc was mainly driven by group differences in approach $>$ avoid soft-drink cues rather than approach $>$ avoid-alcohol cues. That is, patients showed significantly lower activity in the MPFC and NAcc when approaching $>$ avoiding soft drinks, but there were no significant between-group differences in BOLD responses when approaching $>$ avoiding alcohol. It is, however, difficult to interpret the separate approach tendency contrasts in isolation from the alcohol-approach bias interaction of drink type (alcohol $v s$ soft drinks) $\times$ movement (approach $v s$ avoid). Namely, there are methodological reasons to include a neutral category (soft drinks) to the main analysis. First, this allowed us to correct for general approach/avoid tendencies. For example, it may be that patients generally show reduced BOLD responses for approach tendencies of neutral stimuli, such as soft drinks. Second, this corrects for differences in visual feedback and motor movements between approach (zoom in/pull) and avoidance (zoom out/push) trials. Third, defining automatic drug biases as the difference between BOLD signals elicited by drug cues and neutral cues is in line with previous fMRI research on the drug approach bias (Cousijn et al, 2012), the drug attentional bias (Janes et al, 2010; Vollstadt-Klein et al, 2012), and drug-cue reactivity (Beck et al, 2012; Childress et al, 1999; Grusser et al, 2004; Heinz et al, 2004, 2009; Wrase et al, 2007). Nevertheless, the between-group differences in the soft-drink-approach tendency contrast rather than for alcohol may well be meaningful. It may be that the alcohol-approach bias is due to decreased motivational brain responses to naturally rewarding stimuli, such as soft drinks, rather than an increased motivational response to alcohol. This is in line with previous studies showing that addicted individuals demonstrate reduced reward-related activation to naturally rewarding stimuli compared with controls (Volkow et al, 2004; Wrase et al, 2007). In previous fMRI research on drug-approach biases, drug-attentional biases and drug-cue reactivity post-hoc tests exploring interactions have not usually been performed (for an exception, see Braus et al, 2001), nor have plots of the separate $\beta$-coefficients of alcohol/neutral subscores been provided (Beck et al, 2012; Childress et al, 1999; Cousijn et al, 2012; Grusser et al, 2004; Heinz et al, 2004; Janes et al, 2010; Wrase et al, 2007; Vollstadt-Klein et al, 2012). Therefore, future fMRI studies should assess in detail whether increased alcohol cue-evoked reactivity is indeed due to enhanced reactivity to alcohol cues, or rather 
(or additionally) due to reduced reactivity to natural rewards. This could have implications for treatment: rather than attempting to reduce the appeal of alcohol, one could promote the appeal of naturally rewarding stimuli.

A few limitations of the present study need to be mentioned. First, the duration of abstinence of patients varied between 1 week and 6 months, which may have influenced craving and automatic processes. However, length of abstinence was neither negatively correlated with BOLD responses in our ROIs in the approach-alcohol contrast, nor with BOLD responses in the dlPFC in the avoid-alcohol contrast. Therefore, alcohol-approach-biasrelated brain responses may be independent of abstinence and could hence have a significant role in relapse even after long-term abstinence. Second, there were more smokers in the alcohol-dependent group than in the healthy control group. An exploratory analysis revealed that when including smoking behavior as a covariate, mPFC activity did not reach significance ( $p>0.05$, FWE). Hence, it cannot be excluded that the mPFC effects were due to smoking rather than alcohol dependence or to the combination of both addictive behaviors. However, this is unlikely, as our task was exclusively focused on responses to alcohol and soft-drink cues rather than smoking cues. Moreover, smoking behavior was highly correlated with alcohol use and craving in patients. Consequently, including smoking as a covariate may remove variance explained by drinking behavior. Furthermore, although smoking generally influences BOLD (Jacobsen et al, 2002), contrasting approach versus avoid trials made the results independent of general differences in BOLD response due to nicotine use.

In summary, our findings suggest that the automatic alcohol-approach bias is related to changes in the motivational system in alcohol-dependent patients. Even when patients express an explicit wish to remain abstinent, reflexive embodied reactions to alcohol and motivational brain mechanisms are likely to make patients vulnerable for relapse. The findings have implications for treatment of alcohol addiction. Treatment generally focuses on the improvement of conscious control (cognitive behavioral therapy or counseling) and reduction of craving by pharmacotherapy. However, our current results and recent clinical effects of bias modification training (Eberl et al, 2012; Wiers et al, 2011) suggest the automatic drug approach bias as a potential target for clinical intervention. Future studies should focus on whether and how training influences addictive brain states.

\section{FUNDING AND DISCLOSURE}

This study was supported by grants from the German Federal Ministry of Education and Research (BMBF01KR1207C to $\mathrm{AH}$ and $\mathrm{FB}$ ) and the German Research Foundation (DFG_FOR_1617 to AH). CEW: Berlin School of Mind and Brain (Humboldt University Berlin) and Hendrik Muller Vaderlandsch Fonds. CS: Berlin School of Mind and Brain (Humboldt University Berlin). SQP: Berlin School of Mind and Brain (Humboldt University Berlin) and Swiss National Science Foundation. VUL: German National Merit Foundation, the Berlin School of Mind and Brain (Humboldt University Berlin), and the German Academic
Exchange Service. SG: Berlin School of Mind and Brain (Humboldt University Berlin). AH: German Research Foundation (Deutsche Forschungsgemeinschaft; HE 2597/ 4-3; 7-3; 13-1;14-1;15-1; Excellence Cluster Exc 257 \& STE 1430/2-1), German Federal Ministry of Education and Research (01GQ0411; 01QG87164; NGFN Plus 01 GS 08152 and 01 GS 08 159). Unrestricted research grants from Eli Lilly \& Company, Janssen-Cilag, and Bristol-Myers Squibb. JL: DRV-Bund (German Pension Funds), Salus Clinic Lindow (privately owned clinic), IVB (training institute for behaviour therapy), compensation as lecturer at several institutes for behaviour therapy. RWW: Research funding from NWO (Netherlands Science Foundation), ZONMW (Medical Research Counsil Netherlands), EU FP7, ERAB (European Foundation for Alcohol Research), Amsterdam University Funds, Dutch Ministery of Justice and Security, NIDA-NWO. Editor for journal Addiction. Speakers fees/ paid travel from Lundbeck, APS, ERAB, ABMRF, Icana (Yale Imaging Conference), Sucht (German Addiction Society), DFG (German National Science Foundation), Berkeley Harvard, University of Liverpool, University of Padova, University of Rome, University of Missouri, University of Western Australia, European College of Neuropsychopharmacology (ECNP). HW: BMBF (German Ministery of Education), DFG (German Research Foundation), VW (Volkswagen)-foundation, reviewer from the Netherlands Organisation for Scientific Research, speaker from the Humboldt University, WDR (West German Broadcasting), Law Academy of North-Rhine-Westfalia, University of Mainz, The XV Congress of Cognitive Psychotherapy in Milano, editor from the journal Nervenheilkunde. FB: Charité Universitätsmedizin Berlin, German Federal Ministry of Education, Lundbeck, Eli Lilly International Foundation. CKG declares no conflict of interest.

\section{ACKNOWLEDGEMENTS}

We thank Ulrike Malecki, Steffen Pawelczack, Silvia Hoffman, Natalie Becht, and Jana Paeplow for the recruitment of patients from the Salus Clinic Lindow; Heiner Stuke, Amir Javadi, Sven Hädel, Steffen Weissmann, and Scott Stensland for technical support; Nick White, Thomas Gladwin, and Georgina Torbet for proofreading; and Thomas Gladwin for final comments on the manuscript. We thank the Berlin School of Mind and Brain for reimbursement of the joystick.

\section{REFERENCES}

Abrahao KP, Quadros IM, Souza-Formigoni ML (2011). Nucleus accumbens dopamine $\mathrm{D}(1)$ receptors regulate the expression of ethanol-induced behavioural sensitization. Int J Neuropsychopharmacol 14: 175-185.

Baler RD, Volkow ND (2006). Drug addiction: the neurobiology of disrupted self-control. Trends Mol Med 12: 559-566.

Bechara A (2005). Decision making, impulse control and loss of willpower to resist drugs: a neurocognitive perspective. Nat Neurosci 8: 1458-1463.

Beck A, Wustenberg T, Genauck A, Wrase J, Schlagenhauf F, Smolka MN et al (2012). Effect of brain structure, brain function, and brain connectivity on relapse in alcohol-dependent patients. Arch Gen Psychiatry 69: 842-852. 
Brainard DH (1997). The Psychophysics Toolbox. Spat Vis 10: 433-436.

Braus DF, Wrase J, Grusser S, Hermann D, Ruf M, Flor $\mathrm{H}$ et al (2001). Alcohol-associated stimuli activate the ventral striatum in abstinent alcoholics. J Neural Transm 108: 887-894.

Childress AR, Mozley PD, McElgin W, Fitzgerald J, Reivich M, O’Brien CP (1999). Limbic activation during cue-induced cocaine craving. Am J Psychiatry 156: 11-18.

Cousijn J, Goudriaan AE, Ridderinkhof KR, van den Brink W, Veltman DJ, Wiers RW (2012). Approach-bias predicts development of cannabis problem severity in heavy cannabis users: results from a prospective FMRI study. PloS One 7: e42394.

Cousijn J, Goudriaan AE, Wiers RW (2011). Reaching out towards cannabis: approach-bias in heavy cannabis users predicts changes in cannabis use. Addiction 106: 1667-1674.

Deichmann R, Gottfried JA, Hutton C, Turner R (2003). Optimized EPI for fMRI studies of the orbitofrontal cortex. NeuroImage 19(2 Pt 1): 430-441.

Eberl C, Wiers RW, Pawelczack S, Rinck M, Becker ES, Lindenmeyer J (2012). Approach bias modification in alcohol dependence: Do clinical effects replicate and for whom does it work best? Dev Cogn Neurosci.

Ernst LH, Plichta MM, Dresler T, Zesewitz AK, Tupak SV, Haeussinger FB et al (2012). Prefrontal correlates of approach preferences for alcohol stimuli in alcohol dependence. Addict Biol (e-pub ahead of print; doi:10.1111/adb.12005).

Gladwin TE, Figner B, Crone EA, Wiers RW (2011). Addiction, adolescence, and the integration of control and motivation. Dev Cogn Neurosci 1: 364-376.

Grusser SM, Wrase J, Klein S, Hermann D, Smolka MN, Ruf M et al (2004). Cue-induced activation of the striatum and medial prefrontal cortex is associated with subsequent relapse in abstinent alcoholics. Psychopharmacology 175: 296-302.

Hare TA, Camerer CF, Rangel A (2009). Self-control in decisionmaking involves modulation of the vmPFC valuation system. Science 324: 646-648.

Hayashi T, Ko JH, Strafella AP, Dagher A (2013). Dorsolateral prefrontal and orbitofrontal cortex interactions during self-control of cigarette craving. Proc Natl Acad Sci USA 110: 4422-4427.

Heinz A, Beck A, Grusser SM, Grace AA, Wrase J (2009). Identifying the neural circuitry of alcohol craving and relapse vulnerability. Addict Biol 14: 108-118.

Heinz A, Siessmeier T, Wrase J, Hermann D, Klein S, Grusser SM et al (2004). Correlation between dopamine $\mathrm{D}(2)$ receptors in the ventral striatum and central processing of alcohol cues and craving. Am J Psychiatry 161: 1783-1789.

Hyman SE, Malenka RC (2001). Addiction and the brain: the neurobiology of compulsion and its persistence. Nat Rev Neurosci 2: 695-703.

Hyman SE, Malenka RC, Nestler EJ (2006). Neural mechanisms of addiction: the role of reward-related learning and memory. Ann Rev Neurosci 29: 565-598.

Jacobsen LK, Gore JC, Skudlarski P, Lacadie CM, Jatlow P, Krystal JH (2002). Impact of intravenous nicotine on BOLD signal response to photic stimulation. Magn Reson Imaging 20: 141-145.

Janes AC, Pizzagalli DA, Richardt S, Frederick Bde B, Holmes AJ, Sousa J et al (2010). Neural substrates of attentional bias for smoking-related cues: an FMRI study. Neuropsychopharmacology 35: 2339-2345.

Jentsch JD, Taylor JR (1999). Impulsivity resulting from frontostriatal dysfunction in drug abuse: implications for the control of behavior by reward-related stimuli. Psychopharmacology 146: 373-390.

Kahnt T, Heinzle J, Park SQ, Haynes JD (2010). The neural code of reward anticipation in human orbitofrontal cortex. Proc Natl Acad Sci USA 107: 6010-6015.

Kaufman AS, Lichtenberger E (2006). Assessing Adolescent and Adult Intelligence. 3rd edn Wiley: Hoboken, NJ, pp 7.
Koob GF, Volkow ND (2010). Neurocircuitry of addiction. Neuropsychopharmacology 35: 217-238.

Kriegeskorte N, Lindquist MA, Nichols TE, Poldrack RA, Vul E (2010). Everything you never wanted to know about circular analysis, but were afraid to ask. J Cereb Blood Flow Metab 30: $1551-1557$

Love A, James D, Willner P (1998). A comparison of two alcohol craving questionnaires. Addiction 93: 1091-1102.

Maldjian JA, Laurienti PJ, Kraft RA, Burdette JH (2003). An automated method for neuroanatomic and cytoarchitectonic atlas-based interrogation of fMRI data sets. NeuroImage 19: 1233-1239.

Miezin FM, Maccotta L, Ollinger JM, Petersen SE, Buckner RL (2000). Characterizing the hemodynamic response: effects of presentation rate, sampling procedure, and the possibility of ordering brain activity based on relative timing. NeuroImage 11(6 Pt 1): 735-759.

Park SQ, Kahnt T, Beck A, Cohen MX, Dolan RJ, Wrase J et al (2010). Prefrontal cortex fails to learn from reward prediction errors in alcohol dependence. J Neurosci 30: 7749-7753.

Park SQ, Kahnt T, Rieskamp J, Heekeren HR (2011). Neurobiology of value integration: when value impacts valuation. J Neurosci 31: 9307-9314.

Rinck M, Becker ES (2007). Approach and avoidance in fear of spiders. J Behav Therapy Exp Psychiatry 38: 105-120.

Robinson TE, Berridge KC (1993). The neural basis of drug craving: an incentive-sensitization theory of addiction. Brain Res Brain Res Rev 18: 247-291.

Robinson TE, Berridge KC (2003). Addiction. Annu Rev Psychol 54: 25-53.

Roelofs K, Minelli A, Mars RB, van Peer J, Toni I (2009). On the neural control of social emotional behavior. Social Cogn Affective Neurosci 4: 50-58.

Saunders JB, Aasland OG, Babor TF, de la Fuente JR, Grant M (1993). Development of the Alcohol Use Disorders Identification Test (AUDIT): WHO Collaborative project on early detection of persons with harmful alcohol consumption-II. Addiction 88: 791-804.

Schacht JP, Anton RF, Myrick H (2013). Functional neuroimaging studies of alcohol cue reactivity: a quantitative meta-analysis and systematic review. Addict Biol 18: 121-133.

Sheehan DV, Lecrubier Y, Sheehan KH, Amorim P, Janavs J, Weiller E et al (1998). The Mini-International Neuropsychiatric Interview (M.I.N.I.): the development and validation of a structured diagnostic psychiatric interview for DSM-IV and ICD-10. J Clin Psychiatry 59(Suppl 20): 22-33 quiz 34-57.

Shirer WR, Ryali S, Rykhlevskaia E, Menon V, Greicius MD (2012). Decoding subject-driven cognitive states with whole-brain connectivity patterns. Cereb Cortex 22: 158-165.

Skinner HA, Allen BA (1982). Alcohol dependence syndrome: measurement and validation. J Abnormal Psychol 91: 199-209.

Skinner HA, Sheu WJ (1982). Reliability of alcohol use indices. The Lifetime Drinking History and the MAST. J Studies Alcohol 43: 1157-1170.

Spielberger CD, Gorsuch RL, Lushene R, Vagg PR, Jacobs GA (1983). Manual for the State-Trait Anxiety Inventory. Consulting Psychologists Press: Palo Alto, CA.

Stacy AW, Wiers RW (2010). Implicit cognition and addiction: a tool for explaining paradoxical behavior. Ann Rev Clin Psychol 6: 551-575.

Volkow ND, Fowler JS, Wang GJ (2004). The addicted human brain viewed in the light of imaging studies: brain circuits and treatment strategies. Neuropharmacology 47(Suppl 1): 3-13.

Vollstadt-Klein S, Loeber S, Richter A, Kirsch M, Bach P, von der Goltz C et al (2012). Validating incentive salience with functional magnetic resonance imaging: association between mesolimbic 
cue reactivity and attentional bias in alcohol-dependent patients. Addiction Biol 17: 807-816.

Volman I, Toni I, Verhagen L, Roelofs K (2011). Endogenous testosterone modulates prefrontal-amygdala connectivity during social emotional behavior. Cereb Cortex 21: 2282-2290.

Wiers CE, Kühn S, Javadi AH, Korucuoglu O, Wiers RW, Walter H et al (2013). Automatic approach bias towards smoking cues is present in smokers but not in ex-smokers. Psychopharmacology 229: $187-197$.

Wiers RW, Eberl C, Rinck M, Becker ES, Lindenmeyer J (2011). Retraining automatic action tendencies changes alcoholic patients' approach bias for alcohol and improves treatment outcome. Psychol Sci 22: 490-497.

Wrase J, Grusser SM, Klein S, Diener C, Hermann D, Flor H et al (2002). Development of alcohol-associated cues and cue-induced brain activation in alcoholics. Eur Psychiatry 17: 287-291.

Wrase J, Schlagenhauf F, Kienast T, Wustenberg T, Bermpohl F, Kahnt $\mathrm{T}$ et al (2007). Dysfunction of reward processing correlates with alcohol craving in detoxified alcoholics. NeuroImage 35: 787-794.

Zhou Y, Li X, Zhang M, Zhang F, Zhu C, Shen M (2012). Behavioural approach tendencies to heroin-related stimuli in abstinent heroin abusers. Psychopharmacology 221: 171-176.

Supplementary Information accompanies the paper on the Neuropsychopharmacology website (http://www.nature.com/npp) 